PROCEEDINGS OF THE

AMERICAN MATHEMATICAL SOCIETY

Volume 131, Number 5, Pages 1501-1507

S 0002-9939(02)06723-0

Article electronically published on September 19, 2002

\title{
COMMUTATOR ESTIMATES
}

\author{
MICHAEL TAYLOR
}

(Communicated by Andreas Seeger)

\begin{abstract}
We produce a family of commutator estimates, bridging two sharp classical cases of Calderon-Coifman-Meyer type and of Kato-Ponce-Moser type, respectively. The result provides a useful sharpening of other commonly used commutator estimates.
\end{abstract}

\section{INTRODUCTION}

Commutator estimates have played important roles in PDE in a variety of contexts, ranging from energy estimates for hyperbolic PDE and other evolution equations to local and microlocal regularity and propagation of singularities. Here we survey several types of commutator estimates involving pseudodifferential operators, and we produce sharpenings of some of these estimates, in a treatment that unifies them with other commutator estimates.

One basic example is the following.

Proposition 1.1. Assume $1<p<\infty$. Given $P \in O P S_{1,0}^{0}$,

$$
\|[P, f] u\|_{H^{s, p}} \leq C\|f\|_{H^{\sigma, p}}\|u\|_{H^{s-1, p}}
$$

provided

$$
\sigma>\frac{n}{p}+1, \quad 0 \leq s \leq \sigma .
$$

Here $f$ and $u$ are defined on $\mathbb{R}^{n}$. The estimate (1.1) also holds for $P \in O P S_{1, \delta}^{0}, \delta \in$ $[0,1)$, and furthermore for $P \in O P \mathcal{B} S_{1,1}^{0}$. By definition, an element $p(x, D)$ of $O P S_{1, \delta}^{m}$ has symbol $p(x, \xi)$, satisfying

$$
\left|D_{x}^{\beta} D_{\xi}^{\alpha} p(x, \xi)\right| \leq C_{\alpha \beta}(1+|\xi|)^{m-|\alpha|+\delta|\beta|} .
$$

The class $O P \mathcal{B} S_{1,1}^{m}$ consists of operators with symbol $p(x, \xi) \in S_{1,1}^{m}$, satisfying

$$
\operatorname{supp} \hat{p}(\eta, \xi) \subset\{(\eta, \xi):|\eta| \leq \rho|\xi|\},
$$

for some $\rho<1$. This class was introduced by $|\mathrm{Mey}|$ and contains the paradifferential operators introduced in $\left[\mathrm{B}\right.$. We note that $O P \mathcal{B} S_{1,1}^{m}$ contains all the operator classes $O P S_{1, \delta}^{m}, \delta<1$, at least modulo smoothing operators. For a recent example of the use of Proposition 1.1] with $p=2$, see [MS].

Received by the editors December 13, 2001.

2000 Mathematics Subject Classification. Primary 35S05, 35S50.

The author was partially supported by NSF grant DMS-9877077.

(C)2002 American Mathematical Society 
Here is another commutator estimate:

Proposition 1.2. Assume $1<p<\infty$ and $s \geq 0$. Given $P \in O P \mathcal{B} S_{1,1}^{m}, m>0$, we have

$$
\|[P, f] u\|_{H^{s, p}} \leq C\|f\|_{\operatorname{Lip}^{1}}\|u\|_{H^{s+m-1, p}}+C\|f\|_{H^{s+m, p}}\|u\|_{L^{\infty}} .
$$

In case $P \in O P B S_{1,1}^{0}$, we have

$$
\|[P, f] u\|_{H^{s, p}} \leq C\|f\|_{\operatorname{Lip}^{1}}\|u\|_{H^{s-1, p}}+C\|f\|_{H^{s, p}}\left(\|u\|_{L^{\infty}}+\|P u\|_{L^{\infty}}\right) .
$$

Such an estimate was established in $\left[\mathrm{KP}\right.$ for $P=(I-\Delta)^{m / 2}$, in $[\mathrm{T}$ for $P \in$ $O P S_{1,0}^{m}$, and in [AT] for $P \in O P \mathcal{B} S_{1,1}^{m}$. In case $P$ is a differential operator of order $m$, (1.5) is a classical Moser estimate.

The following commutator estimates involve only the Lipschitz norm of $f$.

Proposition 1.3. Assume $1<p<\infty$. Then

$$
P \in O P B S_{1,1}^{1} \Rightarrow\|[P, f] u\|_{L^{p}} \leq C\|f\|_{\operatorname{Lip}^{1}}\|u\|_{L^{p}} .
$$

Also

$$
P \in O P \mathcal{B} S_{1,1}^{0} \Rightarrow\|[P, f] u\|_{H^{s, p}} \leq C\|f\|_{\operatorname{Lip}^{1}}\|u\|_{H^{s-1, p}}, \quad 0 \leq s \leq 1 .
$$

The result (1.7) was proven in Cal for classical first-order pseudodifferential operators, in [CM2] for $P \in O P S_{1,0}^{1}$, and in [AT] for $P \in O P \mathcal{B} S_{1,1}^{1}$. The result (1.8) was proven in [T] for $P \in O P S_{1,0}^{0}$ and in [AT] for $P \in O P \mathcal{B} S_{1,1}^{1}$.

Clearly Proposition 1.2 is stronger than Proposition 1.1 when $s>n / p+1$, and Proposition 1.3 is stronger than Proposition 1.1 when $0 \leq s \leq 1$. One of our goals here is to produce sharpened versions of Proposition 1.1 for the entire range $s \in \mathbb{R}^{+}$. One result we will establish is the following.

Proposition 1.4. Assume $1<p<\infty$. Let $P \in O P \mathcal{B} S_{1,1}^{0}$. Then, for $s>1$, $0<\theta<1$, we have

$$
\|[P, f] u\|_{H^{s, p}} \leq C_{1}\|f\|_{\operatorname{Lip}^{1}}\|u\|_{H^{s-1, p}}+C_{2}\|f\|_{H^{s, p / \theta}}\|u\|_{L^{p /(1-\theta)}},
$$

with $C_{1}=C_{1}(s, p), C_{1}=C_{2}(s, p, \theta)$.

The outline of the rest of this paper is as follows. In $\$ 2$ we discuss a general setup for estimating $[P, f] u$ in terms of a paraproduct decomposition. Given $P \in$ $O P \mathcal{B} S_{1,1}^{m}$, we estimate the $H^{s, p}$-norm of this quantity by $C\|f\|_{\operatorname{Lip}^{1}}\|u\|_{H^{s+m-1, p}}$ plus Sobolev norms of a pair of paraproducts. In $\$ 3$ we discuss various paraproduct estimates to be brought to bear on these remainder terms. In the course of doing this we recall the proof of Proposition 1.2 and we extend Proposition 1.3 to treat $P \in O P \mathcal{B} S_{1,1}^{m}$, with $|m| \leq 1$. In $\$ 4$ we prove Proposition 1.4 and analogues for $P \in O P B S_{1,1}^{m}$, with $m>0$. We show explicitly how certain choices of $\theta$ lead to results that sharpen Proposition 1.1 .

\section{First Basic estimate}

In this section we establish the following proposition, which distills part of the commutator analysis as presented in $\S 3.6$ of $[\mathrm{T}]$. 
Proposition 2.1. Given $P \in O P \mathcal{B} S_{1,1}^{m}, s \geq 0, s \geq-m, 1<p<\infty$, we have

$$
\|[P, f] u\|_{H^{s, p}} \leq C\|f\|_{\operatorname{Lip}^{1}}\|u\|_{H^{s+m-1, p}}+C\left\|T_{u} f\right\|_{H^{s+m, p}}+\left\|T_{P u} f\right\|_{H^{s, p}} .
$$

Proof. We start with expansions

$$
\begin{aligned}
f P u & =T_{f} P u+T_{P u} f+R(f, P u), \\
P(f u) & =P T_{f} u+P T_{u} f+P R(f, u),
\end{aligned}
$$

where $T_{f}$ is Bony's paraproduct operation. Next, we have

$$
f \in \operatorname{Lip}^{1} \Rightarrow\left[T_{f}, P\right] \in O P B S_{1,1}^{m-1},
$$

modulo a smoothing operator. For $P \in O P S_{1,0}^{m}$, this is established in [T], (3.6.4), and for $P \in O P \mathcal{B} S_{1,1}^{m}$, in [AT], Proposition 4.2 (with certain technical adjustments, discussed there). Hence

$$
\left\|\left[P, T_{f}\right] u\right\|_{H^{s, p}} \leq C\|f\|_{\mathrm{Lip}^{1}}\|u\|_{H^{s+m-1, p}} .
$$

Also, Proposition 3.5.D of [T] gives

$$
\|R(f, g)\|_{H^{\sigma, p}} \leq C\|f\|_{\operatorname{Lip}^{1}}\|g\|_{H^{\sigma-1, p}}, \quad \sigma \geq 0,1<p<\infty,
$$

so we have

$$
\|R(f, P u)\|_{H^{s, p}}+\|P R(f, u)\|_{H^{s, p}} \leq C\|f\|_{\operatorname{Lip}^{1}}\|u\|_{H^{s+m-1, p}},
$$

provided $s \geq 0$ and $s+m \geq 0$. This establishes (2.1).

\section{Estimates ON $T_{v} f$}

To apply (2.1), we need estimates on $T_{u} f$ and on $T_{P u} f$. We collect a number of such useful estimates here. To begin, we have

$$
v \in L^{\infty} \Rightarrow T_{v} \in O P \mathcal{B} S_{1,1}^{0}
$$

(cf. [T], (3.5.5)), and hence

$$
\left\|T_{v} f\right\|_{H^{\sigma, p}} \leq C\|v\|_{L^{\infty}}\|f\|_{H^{\sigma, p}}, \quad \sigma \in \mathbb{R}, 1<p<\infty .
$$

Note that this plus (2.1) yields the estimate (1.6). Next we have, for $m>0$,

$$
v \in C_{*}^{-m} \Rightarrow T_{f} \in O P \mathcal{B} S_{1,1}^{m},
$$

where $C_{*}^{-m}$ is a Zygmund space; cf. [T], (3.5.7). Hence

$$
\left\|T_{v} f\right\|_{H^{s, p}} \leq C\|v\|_{C_{*}^{-m}}\|f\|_{H^{s+m, p}}, \quad s \in \mathbb{R}, 1<p<\infty, m>0 .
$$

This plus (2.1) yields the estimate (1.5).

Next, we have the fundamental paraproduct estimate of [CM]:

$$
\left\|T_{v} f\right\|_{L^{p}} \leq C\|v\|_{L^{p}}\|f\|_{\mathrm{BMO}} .
$$

See also [T], Appendix D, for a proof. As shown in [T], Proposition 3.5.F, one can deduce from (3.5) that

$$
\left\|T_{v} f\right\|_{H^{s, p}} \leq C\|v\|_{H^{s-r, p}}\|f\|_{\mathfrak{h}^{r, \infty}}, \quad 0 \leq s \leq r,
$$

for $r \in \mathbb{Z}^{+}$. Here $\mathfrak{h}^{r, \infty}$ denotes the bmo-Sobolev space

$$
\mathfrak{h}^{r, \infty}=(I-\Delta)^{-r / 2} \text { bmo, }
$$


and bmo denotes the localized John-Nirenberg space. The case $r=1$ of (3.6) is particularly germane to our analysis. This estimate applied to the remainders in (2.1) yields the following extension of Proposition 1.3

Proposition 3.1. Given $P \in O P B S_{1,1}^{m},|m| \leq 1$, we have

$$
\|[P, f] u\|_{H^{s, p}} \leq C\|f\|_{\operatorname{Lip}^{1}}\|u\|_{H^{s+m-1, p}},
$$

provided either

$$
0 \leq m \leq 1 \text { and } 0 \leq s \leq 1-m, \quad \text { or } \quad-1 \leq m \leq 0 \text { and }|m| \leq s \leq 1 .
$$

Proof. We have

$$
\begin{aligned}
0 \leq s \leq 1 \Rightarrow\left\|T_{P u} f\right\|_{H^{s, p}} & \leq C\|P u\|_{H^{s-1, p}}\|f\|_{\mathfrak{h}^{1, \infty}} \\
& \leq C\|u\|_{H^{s+m-1, p}}\|f\|_{\mathfrak{h}^{1, \infty}}
\end{aligned}
$$

and

$$
0 \leq s+m \leq 1 \Rightarrow\left\|T_{u} f\right\|_{H^{s+m, p}} \leq C\|u\|_{H^{s+m-1, p}}\|f\|_{\mathfrak{h}^{1, \infty}},
$$

from which the result follows.

Next, we interpolate between (3.2) and (3.5) to obtain further useful estimates.

Proposition 3.2. For $1<p<\infty, s \in \mathbb{R}, 0<\theta \leq 1$,

$$
\left\|T_{v} f\right\|_{H^{s, p}} \leq C\|v\|_{L^{p /(1-\theta)}}\|f\|_{H^{s, p / \theta}}
$$

Proof. If $v(z)$ and $f(z)$ are holomorphic on $\Omega=\{z: 0<\operatorname{Re} z<1\}$, appropriately bounded on $\bar{\Omega}$, with $v(i y)$ bounded in $L^{p}, v(1+i y)$ bounded in $L^{\infty}, f(i y)$ bounded in bmo, and $f(1+i y)$ bounded in $H^{\sigma, p}$, we have for $\Phi(z)=T_{v(z)} f(z)$ that $\Phi(\theta) \in$ $H^{\theta \sigma, p}$. Results of [FS] yield [bmo, $\left.H^{\sigma, p}\right]_{\theta}=H^{\theta \sigma, p / \theta}$, so we have (3.11).

\section{Further COMmutator estimates}

To begin, we record how Proposition 1.1 is a consequence of (1.6) and (1.8).

Proof of Proposition 1.1. Fix $\sigma>n / p+1$. To prove (1.1), it suffices to establish it for the two endpoint cases, $s=0$ and $s=\sigma$; the rest follows by interpolation. But (1.6) implies (1.1) for $s=\sigma$ and (1.8) implies (1.1) for $s=0$.

To be sure, this is a sledgehammer approach to Proposition 1.1 A direct paraproduct proof is briefly presented in [MS] (with specific reference to $p=2$ ). We redeem our approach by now presenting the

Proof of Proposition 1.4. In light of (2.1), it suffices to show that, for $0<\theta<1$,

$$
\left\|T_{u} f\right\|_{H^{s, p}}+\left\|T_{P u} f\right\|_{H^{s, p}} \leq C\|f\|_{H^{s, p / \theta}}\|u\|_{L^{p /(1-\theta)}},
$$

given $P \in O P \mathcal{B} S_{1,1}^{0}$. But note that (3.11) then applies to $v=u$ and to $v=P u$, so we are done.

We now discuss in more detail how Proposition 1.4 refines Proposition 1.1

Proposition 4.1. Given $\sigma=n / p+1$ and $1<s<\sigma$, there exists $\theta \in(0,1)$ such that

$$
\|f\|_{H^{s, p / \theta}} \leq C\|f\|_{H^{\sigma, p}} \quad \text { and } \quad\|u\|_{L^{p /(1-\theta)}} \leq C\|u\|_{H^{s-1, p}} .
$$


Proof. For $s=1$ and $s=\sigma$, respectively, we have

$$
\begin{array}{rlrl}
H^{\sigma, p} \subset \mathfrak{h}^{1, \infty}, & & H^{0, p}=L^{p}, \\
H^{\sigma, p} & =H^{\sigma, p}, & & H^{\sigma-1, p} \subset \text { bmo } .
\end{array}
$$

Interpolation gives

$$
H^{\sigma, p} \subset H^{\theta(\sigma-1)+1, p / \theta}, \quad H^{\theta(\sigma-1), p} \subset L^{p /(1-\theta)} .
$$

Alternatively (and with less technology), the results of (4.4) follow directly from the Sobolev embedding theorem. As $\theta$ runs over $(0,1), s=\theta(\sigma-1)+1$ runs over $(1, \sigma)$, so we have (4.2).

Note that, if $\theta=\theta(n, p, s) \in(0,1)$ is picked so that (4.2) holds, then $p / \theta=$ $n /(s-1)$, and we have

$$
\|[P, f] u\|_{H^{s, p}} \leq C\left(\|f\|_{\mathrm{Lip}^{1}}+\|f\|_{H^{s, n /(s-1)}}\right)\|u\|_{H^{s-1, p}}, \quad 1<s<\frac{n}{p}+1,
$$

for $P \in O P \mathcal{B} S_{1,1}^{0}$, which is sharper than (1.1) in this range. The estimate (4.5) is a corollary of the following even sharper result, a special case of (1.9):

$$
\|[P, f] u\|_{H^{s, p}} \leq C_{1}\|f\|_{\operatorname{Lip}^{1}}\|u\|_{H^{s-1, p}}+C_{2}\|f\|_{H^{s, n /(s-1)}}\|u\|_{L^{n p /(n-(s-1) p)}},
$$

valid for $1<s<n / p+1$.

As already noted, (1.6) is sharper than (1.1) when $s>n / p+1$. We next sharpen (1.1) in the case $s=n / p+1$. In such a case we have

$$
u, P u \in H^{n / p, p} \subset \text { bmo } \subset C_{*}^{0} .
$$

Now (3.4) fails for $m=0$, but a simple modification of the Littlewood-Paley argument used in such estimates gives

$$
\left\|T_{u} g\right\|_{H^{s, p}} \leq C\|(\log \Lambda) g\|_{H^{s, p}}\|u\|_{C_{*}^{0}}
$$

for $s \in \mathbb{R}, 1<p<\infty$, where

$$
\Lambda=(2 I-\Delta)^{1 / 2} .
$$

This estimate together with (2.1) yields

$$
\|[P, f] u\|_{H^{n / p+1, p}} \leq C\|f\|_{\operatorname{Lip}^{1}}\|u\|_{H^{n / p, p}}+C\|(\log \Lambda) f\|_{H^{n / p+1, p}}\|u\|_{C_{*}^{0}},
$$

for $P \in O P B S_{1,1}^{0}$.

We now derive further estimates on $\|[P, f] u\|_{H^{s, p}}$ when $P \in O P \mathcal{B} S_{1,1}^{m}$ with $m>$ 0 . In all cases we retain the standing assumption that

$$
s \geq 0, \quad 1<p<\infty .
$$

The estimates we need derive from estimates on $\left\|T_{u} f\right\|_{H^{s+m, p}}$ and on $\left\|T_{P u} f\right\|_{H^{s, p}}$. Thus various cases arise, depending on whether $s+m \in(1, n / p+1)$ or not and on whether $s \in(1, n / p+1)$ or not. We break the first issue into four cases:

$$
\begin{aligned}
s+m & >\frac{n}{p}+1, \\
s+m & =\frac{n}{p}+1, \\
1<s+m & <\frac{n}{p}+1, \\
0 \leq s+m & \leq 1 .
\end{aligned}
$$

In Case (I) the estimate (1.5) is always a servicable sharpening of

$$
\|[P, f] u\|_{H^{s, p}} \leq C\|f\|_{H^{\sigma, p}}\|u\|_{H^{s+m-1, p}},
$$


for $n / p+1<s+m \leq \sigma$. Case (II) calls for the estimate

$$
\left\|T_{u} f\right\|_{H^{s+m, p}} \leq C\|(\log \Lambda) f\|_{H^{n / p+1, p}}\|u\|_{C_{*}^{0}} .
$$

Following the arguments of Proposition 4.1, we see that Case (III) calls for the estimate

$$
\left\|T_{u} f\right\|_{H^{s+m, p}} \leq C\|f\|_{H^{s+m, n /(s+m-1)}}\|u\|_{L^{n p /(n-(s+m-1) p)},}
$$

and that the right side of (4.14) is dominated by

$$
C\|f\|_{H^{n / p+1, p}}\|u\|_{H^{s+m-1, p}} .
$$

Case (IV) calls for the estimate

$$
\left\|T_{u} f\right\|_{H^{s+m, p}} \leq C\|f\|_{\mathfrak{h}^{1, \infty}}\|u\|_{H^{s+m-1, p}},
$$

which follows from (3.6), with $r=1$. The right side of (4.16) is also dominated by (4.15).

Cases (II)-(IV) break up into sub-cases in which to estimate $\left\|T_{P u} f\right\|_{H^{s, p}}$. We have

$$
\begin{aligned}
& 1<s<\frac{n}{p}+1, \\
& 0 \leq s \leq 1 .
\end{aligned}
$$

Note that, since we are assuming $m>0$, the case $s \geq n / p+1$ is subsumed in Case (I). Now we see that Case (A) calls for the estimate

$$
\left\|T_{P u} f\right\|_{H^{s, p}} \leq C\|f\|_{H^{s, n /(s-1)}}\|u\|_{H^{m, n p /(n-(s-1) p)},},
$$

and, by Proposition 4.1, the right side of (4.17) is dominated by 4.15). Finally, Case (B) calls for the estimate

$$
\left\|T_{P u} f\right\|_{H^{s, p}} \leq C\|f\|_{\mathfrak{h}^{1, \infty}}\|u\|_{H^{s+m-1, p}},
$$

also dominated by (4.15).

In particular, in all cases we have sharpenings of the following generalization of Proposition 1.1

Proposition 4.2. Assume $1<p<\infty, m \geq 0$. Given $P \in O P \mathcal{B} S_{1,1}^{m}$, we have

$$
\|[P, f] u\|_{H^{s, p}} \leq C\|f\|_{H^{\sigma, p}}\|u\|_{H^{s+m-1, p}},
$$

provided

$$
\sigma>\frac{n}{p}+1, \quad s \geq 0, \quad s+m \leq \sigma .
$$

\section{REFERENCES}

[AT] P. Auscher and M. Taylor, Paradifferential operators and commutator estimates, Comm. PDE 20 (1995), 1743-1775. MR 96j:47047

[B] J.-M. Bony, Calcul symbolique et propagation des singularitiés pour les équations aux dérivées nonlinéaires, Ann. Sci. Ecole Norm. Sup. 14 (1981), 209-246. MR 84h:35177

[Cal] A. Calderon, Commutators of singular integral operators, Proc. NAS, USA 53 (1965), 1092-1099. MR 31:1575

[CM] R. Coifman and Y. Meyer, Au dela des opérateurs pseudodifferentiels, Asterisque \#57, Soc. Math. de France, 1978. MR 81b:47061

[CM2] R. Coifman and Y. Meyer, Commutateurs d'intégrales singulières et opérateurs multilinéaires, Ann. Sci. Inst. Fourier 28 (1978), 177-202. MR 80a:47076

[FS] C. Fefferman and E. Stein, $H^{p}$ functions of several variables, Acta Math. 129 (1972), 137-193.

[KP] T. Kato and G. Ponce, Commutator estimates and the Euler and Navier-Stokes equations, CPAM 41 (1988), 891-907. MR 90f:35162 
[MS] G. Métivier and S. Schochet, The incompressible limit of the non-isentropic Euler equations, Arch. Ration. Mech. Anal. 158 (2001), 61-90. MR 2002d:76095

[Mey] Y. Meyer, Remarques sur un théorème de J. M. Bony, Rend. del Circolo Mat. di Palermo (suppl. II:1) (1981), 1-20. MR 83b:35169

[T] M. Taylor, Pseudodifferential Operators and Nonlinear PDE, Birkhauser, Boston, 1991. MR 92j:35193

Department of Mathematics, University of North Carolina, Chapel Hill, North CarOLINA 27599

E-mail address: met@math.unc.edu 